Pertti Mattila $\cdot$ Joan Verdera

\title{
Convergence of singular integrals with general measures
}

Received October 14, 2006 and in revised form June 17, 2007

Abstract. We show that $L^{2}$-bounded singular integrals in metric spaces with respect to general measures and kernels converge weakly. This implies a kind of average convergence almost everywhere. For measures with zero density we prove the almost everywhere existence of principal values.

Keywords. Singular integrals, principal values, martingales

\section{Introduction}

Singular integrals with respect to general measures in $\mathbb{R}^{n}$, and also in metric spaces, have been widely studied (see, e.g., $[\mathrm{C}],[\mathrm{CW}],[\overline{\mathrm{D}}],[\mathrm{DS}],[\mathrm{M}],[\mathrm{P}],[\mathrm{Ve}]$ and $[\mathrm{V}]$ ). In this paper our setting is a separable metric space $(X, d)$ with a finite Borel measure $\mu$ and a Borel measurable antisymmetric kernel $K: X \times X \backslash\{(x, y): x=y\} \rightarrow \mathbb{R}$. Antisymmetry means that

$$
K(x, y)=-K(y, x) \quad \text { for } x, y \in X, x \neq y .
$$

Moreover, we shall assume that $K$ is bounded in $\{(x, y) \in X \times X: d(x, y)>\delta\}$ for every $\delta>0$. We shall also always assume that Vitali's covering theorem is valid for $\mu$ and the family of closed balls. Although this is not automatically true even when $X$ is compact, it is true for example if $X=\mathbb{R}^{n}$ or $\mu$ is doubling (see, e.g., [F, Section 2.8]).

The singular integral operator $T$ associated with $\mu$ and $K$ is formally given by

$$
T(f)(x)=\int K(x, y) f(y) d \mu y .
$$

The problem which appears already in all classical cases such as the Hilbert transform on $\mathbb{R}$, i.e., $K(x, y)=1 /(y-x)$, is that usually this integral does not exist when $x \in \operatorname{spt} \mu$,

P. Mattila: Department of Mathematics and Statistics, P.O. Box 68, FI-00014 University of Helsinki, Finland; e-mail: Pertti.Mattila@Helsinki.fi

J. Verdera: Departament de Matemàtiques, Universitat Autònoma de Barcelona, 08193 Bellaterra, Barcelona, Catalonia; e-mail: jvm@ mat.uab.cat

Mathematics Subject Classification (2000): Primary 42B20 
the support of $\mu$. When $\mu$ is the Lebesgue measure $\mathcal{L}^{n}$ on $\mathbb{R}^{n}$ and $K$ is a standard Calderón-Zygmund kernel, this can be overcome by defining

$$
T(f)(x)=\lim _{\epsilon \rightarrow 0} T_{\epsilon} f(x),
$$

where

$$
T_{\epsilon}(f)(x)=\int_{X \backslash B(x, \epsilon)} K(x, y) f(y) d \mu y .
$$

Here $B(x, \epsilon)$ is the open ball with centre $x$ and radius $\epsilon$. In such a case the limit exists trivially for smooth functions due to cancellations, and by the denseness of smooth functions in $L^{1}\left(\mathcal{L}^{n}\right)$ standard techniques can be used to show that it exists almost everywhere for $L^{1}$-functions $f$. For general measures this approach fails. Unless $\mu$ has strong symmetry properties around points in its support, there are not enough cancellations to guarantee the existence of the limit even for constant functions. However, when $K$ is antisymmetric one often defines $T(f)$ as a distribution by

$$
(T(f), g)=\frac{1}{2} \iint K(x, y)(f(x) g(y)-f(y) g(x)) d \mu x d \mu y
$$

when $f$ and $g$ are bounded Lipschitz functions (see [C] or [D1]).

A central concept in the theory of singular integrals is the boundedness in $L^{2}$. This can be formulated in several ways which all agree in the classical case of Calderón-Zygmund kernels and the Lebesgue measure. One way is to say that the distributionally defined operator $T$, as in (1.2), is bounded in $L^{2}(\mu)$ if it has a bounded extension to $L^{2}(\mu) \rightarrow$ $L^{2}(\mu)$. Another way is to require that the truncated operators $T_{\epsilon}, \epsilon>0$, are uniformly bounded in $L^{2}(\mu)$. This agrees very generally with the boundedness in $L^{2}(\mu)$ of the sublinear maximal operator $T^{*}$ :

$$
T^{*}(f)(x)=\sup _{\epsilon>0}\left|T_{\epsilon}(f)(x)\right|
$$

(see [NTV]).

A natural question is whether the $L^{2}$-boundedness forces the $\operatorname{limit}_{\lim _{\epsilon \rightarrow 0}} T_{\epsilon}(f)(x)$ to exist for $\mu$-almost all $x \in X$. One would expect this to be true at least if $\mu$ is an $m$-dimensional Ahlfors-David-regular measure in $\mathbb{R}^{n}$ :

$$
r^{m} / C \leq \mu(B(x, r)) \leq C r^{m} \quad \text { for } x \in \operatorname{spt} \mu, 0<r<\operatorname{diam}(\operatorname{spt} \mu),
$$

and $K$ is the vector-valued Riesz kernel $|x-y|^{-m-1}(x-y)$. In fact, by a result of Tolsa (see [T1]), this is true when $m=1$ even for much more general measures, but the proof is based on very special relations with the kernel $x /|x|^{2}$ (essentially the Cauchy kernel $1 / z$ for $z \in \mathbb{C}=\mathbb{R}^{2}$ ) and the so-called Menger curvature. We shall discuss some relations of this problem to rectifiability at the end of the paper. And we shall mention some kernels for which $L^{2}$-boundedness does not give the almost everywhere convergence of principal values.

In this paper we prove some substitutes for (1.1) under the $L^{2}$-boundedness: 
1.4. Theorem. Suppose that $T^{*}$ (defined by (1.3)) is bounded in $L^{2}(\mu)$, that is, there exists a constant $C_{0}$ such that

$$
\int T^{*}(f)^{2} d \mu \leq C_{0} \int f^{2} d \mu
$$

for $f \in L^{2}(\mu)$. Then the truncated operators $T_{\epsilon}$ converge weakly in $L^{2}(\mu)$, that is, there exists a bounded linear operator $T: L^{2}(\mu) \rightarrow L^{2}(\mu)$ such that

$$
\lim _{\epsilon \rightarrow 0} \int T_{\epsilon}(f) g d \mu=\int T(f) g d \mu
$$

for $f, g \in L^{2}(\mu)$. Moreover,

$$
T(f)(z)=\lim _{r \rightarrow 0} \frac{1}{\mu(B(z, r))} \int_{B(z, r)}\left(\int_{X \backslash B(z, r)} K(x, y) f(y) d \mu y\right) d \mu x
$$

for $\mu$-almost all $z \in X$.

So even if we do not know that $T(f)$ would exist as the limit of the simpler integrals $T_{\epsilon}(f)$, we know that it is almost everywhere the limit of the more complicated but still concrete integrals of Theorem 1.4

Observe that with some natural estimates the limit operator $T$ satisfies (1.2). This is so if, for example,

$$
\iint \mid K(x, y \mid d(x, y) d \mu y d \mu x<\infty,
$$

as one easily checks. In many cases also the converse in the first part of Theorem 1.4 is true. Namely, by the Banach-Steinhaus theorem the weak convergence implies that the truncated operators $T_{\epsilon}$ are uniformly bounded and, as said before, this is often equivalent to the $L^{2}$-boundedness of $T^{*}$.

We prove Theorem 1.4 in Section 2. We first establish the weak convergence. Then we deduce from it the average convergence using the Lebesgue differentiation theorem. We shall also indicate in Section 3 another way of getting the average convergence via the martingale convergence theorem.

In Section 4 we apply Theorem 1.4 to prove the following result on the existence of principal values for measures with zero density:

1.6. Theorem. Suppose $X=\mathbb{R}^{n}$ or $\mu$ is doubling. Let $h:(0, \infty) \rightarrow(0, \infty)$ be an increasing function such that $\lim _{r \rightarrow 0} h(r)=0$ and $h(2 r) \leq C h(r)$ for $r>0$ and suppose that for $x, y \in X, x \neq y$,

$$
|K(x, y)| \leq \frac{1}{h(d(x, y))},
$$

and for $z \in X, z \neq x$ with $d(x, y)>2 d(y, z)$,

$$
|K(x, y)-K(x, z)| \leq \frac{d(y, z)}{d(x, y) h(d(x, y))} .
$$


Suppose also that for all $x \in X$ and $r>0$,

$$
\mu(B(x, r)) \leq h(r)
$$

and for $\mu$-almost all $x \in X$,

$$
\lim _{r \rightarrow 0} \frac{\mu(B(x, r))}{h(r)}=0 .
$$

If $T^{*}: L^{2}(\mu) \rightarrow L^{2}(\mu)$ is bounded, then for $f \in L^{1}(\mu)$ and for $\mu$-almost all $x \in X$,

$$
\lim _{\epsilon \rightarrow 0} T_{\epsilon}(f)(x)=T(f)(x)
$$

where $T$ is the weak limit operator of Theorem 1.4

Note that originally $T(f)$ was only defined for $f \in L^{2}(\mu)$, but under the assumptions of the theorem it has a unique extension to $L^{1}(\mu)$ because we have the weak $L^{1}$-inequality: for $t>0$,

$$
\mu\left(\left\{x \in X:\left|T^{*}(f)(x)\right|>t\right\}\right) \leq C\|f\|_{1} / t .
$$

For doubling measures in metric spaces this was proved in $[\mathrm{CW}]$ and for general measures in $\mathbb{R}^{n}$ in [NTV]. The assumptions on the kernels in [NTV] are not quite the same as above but it is easy to check that the proofs can be modified.

Quite often the growth condition (1.9) is a consequence of the $L^{2}$-boundedness of $T^{*}$ (see [D1, p. 56]).

For general kernels $K$ as above the assumption 1.10 is necessary, as an example of David, which we discuss at the end of the paper, shows.

A particular but interesting instance of the above result arises in the following situation. We take $X=\mathbb{R}^{n}$ and an underlying measure $\mu$ which satisfies the growth condition $\mu(B(x, r)) \leq C r^{m}$ for each $x$ and each $r>0$. The kernel is a standard smooth antisymmetric $m$-dimensional kernel satisfying the usual conditions

$$
|K(x, y)| \leq \frac{1}{|x-y|^{m}},
$$

and

$$
|K(x, y)-K(x, z)| \leq \frac{|y-z|}{|x-y|^{m+1}}, \quad|x-y|>2|y-z| .
$$

Then (1.10) says that $m$-dimensional density vanishes for $\mu$-almost all $x$, namely,

$$
\lim _{r \rightarrow 0} \frac{\mu(B(x, r))}{r^{m}}=0 .
$$

This, of course, excludes $m$-dimensional Ahlfors-David regular sets. See the remarks in Section 5.

\section{Proof of Theorem 1.4}

Let $B$ be a closed ball in $X$. We denote by $\chi_{A}$ the characteristic function of a set $A$ and by $A^{c}$ its complement in $X$. We have, for all $\epsilon>0$ (1 denotes the constant function identically 1), 


$$
\int T_{\epsilon}(1) \chi_{B} d \mu=-\int T_{\epsilon}\left(\chi_{B}\right) d \mu=-\int_{B^{c}} T_{\epsilon}\left(\chi_{B}\right) d \mu,
$$

because by antisymmetry

$$
\int_{B} T_{\epsilon}\left(\chi_{B}\right) d \mu=0 .
$$

Clearly, for all $x \in B^{c}$, (since $B$ is closed) the limit

$$
T\left(\chi_{B}\right)(x):=\lim _{\epsilon \rightarrow 0} T_{\epsilon}\left(\chi_{B}\right)(x)
$$

exists. As $\left|T_{\epsilon}\left(\chi_{B}\right)\right| \leq T^{*}\left(\chi_{B}\right) \in L^{1}(\mu)$, the dominated convergence theorem yields

$$
\lim _{\epsilon \rightarrow 0} \int T_{\epsilon}(1) \chi_{B} d \mu=-\lim _{\epsilon \rightarrow 0} \int_{B^{c}} T_{\epsilon}\left(\chi_{B}\right) d \mu=-\int_{B^{c}} T\left(\chi_{B}\right) d \mu .
$$

Let $S$ be the dense subspace of $L^{2}(\mu)$ consisting of the finite linear combinations of the characteristic functions of closed balls. (It is easy to verify that $S$ is dense since we have assumed Vitali's covering theorem for $\mu$.) Fix $f$ in $L^{2}(\mu)$ and take $b$ in $S$ extremely close to $f$ in $L^{2}(\mu)$. Then for $0<\epsilon<\delta$,

$$
\int\left(T_{\delta}(1)-T_{\epsilon}(1)\right) f d \mu=\int\left(T_{\delta}(1)-T_{\epsilon}(1)\right)(f-b) d \mu+\int\left(T_{\delta}(1)-T_{\epsilon}(1)\right) b d \mu .
$$

By 2.1 , the second term goes to 0 as $\delta \rightarrow 0$. For the first term we have, by the Schwarz inequality and the $L^{2}$-boundedness $(1.5)$ of $T^{*}$,

$$
\begin{aligned}
\left|\int\left(T_{\delta}(1)-T_{\epsilon}(1)\right)(f-b) d \mu\right| & \leq\left\|T_{\delta}(1)-T_{\epsilon}(1)\right\|_{2}\|f-b\|_{2} \\
& \leq 2\left\|T^{*}(1)\right\|_{2}\|f-b\|_{2} \leq 2\left(C_{0} \mu(X)\right)^{1 / 2}\|f-b\|_{2},
\end{aligned}
$$

which we can make as small as we want. This implies that the limit

$$
\lim _{\epsilon \rightarrow 0} \int T_{\epsilon}(1) f d \mu
$$

exists and is finite for all $f \in L^{2}(\mu)$.

Let again $B$ be a closed ball and $f \in L^{2}(\mu)$. Then for $\epsilon>0$,

$$
\begin{aligned}
\int T_{\epsilon}\left(\chi_{B}\right) f d \mu= & \int_{B} \int_{B \backslash B(x, \epsilon)} K(x, y) d \mu y f(x) d \mu x \\
& +\int_{B^{c}} \int_{B \backslash B(x, \epsilon)} K(x, y) d \mu y f(x) d \mu x .
\end{aligned}
$$

Applying what we proved above to the measure $\chi_{B} \mu$ we conclude that the first integral converges as $\epsilon \rightarrow 0$. The second integral converges again by the dominated convergence theorem, since

$$
\left|\int_{B \backslash B(x, \epsilon)} K(x, y) d \mu y f(x)\right| \leq T^{*}\left(\chi_{B}\right)(x)|f(x)|
$$


and $T^{*}\left(\chi_{B}\right) f \in L^{1}(\mu)$. Then also

$$
\lim _{\epsilon \rightarrow 0} \int T_{\epsilon}(b) f d \mu
$$

exists for all $f \in L^{2}(\mu), b \in S$. Arguing as above with the $L^{2}$-boundedness we find that

$$
\lim _{\epsilon \rightarrow 0} \int T_{\epsilon}(g) f d \mu
$$

exists for all $f, g \in L^{2}(\mu)$. This implies easily that there exists a bounded linear operator $T: L^{2}(\mu) \rightarrow L^{2}(\mu)$ such that

$$
\int T(g) f d \mu=\lim _{\epsilon \rightarrow 0} \int T_{\epsilon}(g) f d \mu
$$

for all $f, g \in L^{2}(\mu)$, and we have established the required weak convergence.

Let $B=B(z, r)$ be an open ball with $\mu(B)>0$. Using the antisymmetry of $K$ we have, for all $\epsilon>0$,

$$
\begin{aligned}
\int_{B} T_{\epsilon}\left(f \chi_{B^{c}}\right) d \mu & =-\int f \chi_{B^{c}} T_{\epsilon}\left(\chi_{B}\right) d \mu=-\int f T_{\epsilon}\left(\chi_{B}\right) d \mu+\int f \chi_{B} T_{\epsilon}\left(\chi_{B}\right) d \mu \\
& =\int_{B} T_{\epsilon}(f) d \mu+\int_{B}\left(f-f_{B}\right) T_{\epsilon}\left(\chi_{B}\right) d \mu,
\end{aligned}
$$

where $f_{B}=\frac{1}{\mu(B)} \int_{B} f d \mu$ and $\int_{B} T_{\epsilon}\left(\chi_{B}\right) d \mu=0$. Letting $\epsilon \rightarrow 0$, we obtain, for the weak limit operator $T$,

$$
\int_{B} T\left(f \chi_{B^{c}}\right) d \mu=\int_{B} T(f) d \mu+\int_{B}\left(f-f_{B}\right) T\left(\chi_{B}\right) d \mu .
$$

Dividing by $\mu(B)=\mu(B(z, r))$ and letting $r \rightarrow 0$, we have for $\mu$-almost all $z$ for the first term of the right hand side, by the Lebesgue differentiation theorem,

$$
\lim _{r \rightarrow 0} \frac{1}{\mu(B(z, r))} \int_{B(z, r)} T(f) d \mu=T(f)(z),
$$

and for the second term, by the Schwarz inequality, $L^{2}$-boundedness of $T$ and the Lebesgue differentiation theorem,

$$
\lim _{r \rightarrow 0} \frac{1}{\mu(B(z, r))} \int_{B(z, r)}\left(f-f_{B(z, r)}\right) T\left(\chi_{B(z, r)}\right) d \mu=0 .
$$

On the other hand,

$$
T_{\epsilon}\left(f \chi_{B^{c}}\right)(x)=\int_{B^{c} \backslash B(x, \epsilon)} K(x, y) f(y) d \mu y \rightarrow \int_{B^{c}} K(x, y) f(y) d \mu y
$$


as $\epsilon \rightarrow 0$ for $x \in B$ with $\left|T_{\epsilon}\left(f \chi_{B^{c}}\right)(x)\right| \leq\left|T^{*}\left(f \chi_{B^{c}}\right)(x)\right|$, and so by the dominated convergence theorem,

$$
\int_{B} T\left(f \chi_{B^{c}}\right) d \mu=\lim _{\epsilon \rightarrow 0} \int_{B} T_{\epsilon}\left(f \chi_{B^{c}}\right) d \mu=\int_{B} \int_{B^{c}} K(x, y) f(y) d \mu y d \mu x .
$$

Combining the above equations, we obtain

$$
\lim _{r \rightarrow 0} \frac{1}{\mu(B(z, r))} \int_{B(z, r)} \int_{B(z, r)^{c}} K(x, y) f(y) d \mu y d \mu x=T f(z)
$$

for $\mu$-almost all $z \in X$. This proves the theorem.

For further reference we record that for every ball $B$,

$$
\int_{B} T(1) d \mu=\int_{B} T\left(\chi_{B^{c}}\right) d \mu=-\int_{B^{c}} T\left(\chi_{B}\right) d \mu,
$$

which follows by antisymmetry.

\section{Martingales}

We introduce a general nested system of sets. Standard examples are dyadic lattices of cubes in $\mathbb{R}^{n}$. For each $k \in \mathbb{N}=\{1,2, \ldots\}$ let $\mathcal{D}_{k}$ be a countable disjoint partition of $X$ into $\mu$-measurable sets $D$ such that $\mu(\partial D)=0$. Let $\mathcal{D}=\bigcup_{k=1}^{\infty} \mathcal{D}_{k}$. We assume that the system $\left\{\mathcal{D}_{k}\right\}$ is nested in the sense that every $D \in \mathcal{D}_{k+1}$ is contained in some $D^{\prime} \in \mathcal{D}_{k}$. Then every $D^{\prime} \in \mathcal{D}_{k}$ is a disjoint union of sets in $\mathcal{D}_{k+1}$.

Suppose that $T^{*}$ is bounded in $L^{2}(\mu)$. Let $f \in L^{2}(\mu)$ and $D \in \mathcal{D}_{k}$. As $\mu(\partial D)=0$ we have, for $\mu$-almost all $x \in D$,

$$
\int_{D^{c}} K(x, y) f(y) d \mu y=\lim _{\epsilon \rightarrow 0} \int_{D^{c} \backslash B(x, \epsilon)} K(x, y) f(y) d \mu y .
$$

Moreover,

$$
\left|\int_{D^{c} \backslash B(x, \epsilon)} K(x, y) f(y) d \mu y\right| \leq T^{*}\left(f \chi_{D^{c}}\right)(x) \leq T^{*}(f)(x)+T^{*}\left(f \chi_{D}\right)(x) .
$$

If also $g \in L^{2}(\mu)$, by the dominated convergence theorem we get

$$
\begin{aligned}
\int_{D}\left|\int_{D^{c}} K(x, y) f(y) d \mu y g(x)\right| & d \mu x \\
\leq & \int_{D} T^{*}(f)|g| d \mu+\int_{D} T^{*}\left(f \chi_{D}\right)|g| d \mu<\infty .
\end{aligned}
$$

Suppose now in addition that $f$ is non-negative. Then by 3.1 we can define, for $k \in \mathbb{N}$,

$$
S_{k} f(z)=\left(\int_{D} f d \mu\right)^{-1} \int_{D} \int_{D^{c}} K(x, y) f(y) d \mu y f(x) d \mu x
$$

when $z \in D \in \mathcal{D}_{k}$, where we interpret $S_{k} f(z)$ as 0 when $z \in D \in \mathcal{D}_{k}$ and $\int_{D} f d \mu=0$. 
Let $v$ be the finite Borel measure on $X$ such that

$$
v(B)=\int_{B} f d \mu
$$

for Borel sets $B \subset X$. Let $\mathcal{A}_{k}$ be the $\sigma$-algebra generated by $\mathcal{D}_{k}$. We shall check that $\left(S_{k} f, \mathcal{A}_{k}\right)$ is a martingale (with respect to $v$ ).

Let $D \in \mathcal{D}_{k}$ and let $D_{1}, D_{2}, \ldots$ be sets in $\mathcal{D}_{k+1}$ which form a disjoint partition of $D$. Then

$$
\begin{aligned}
\int_{D} S_{k+1} f d v & =\sum_{i} \int_{D_{i}} S_{k+1} f d \nu=\sum_{i} \int_{D_{i}} \frac{1}{\nu\left(D_{i}\right)} \int_{D_{i}} \int_{D_{i}^{c}} K(x, y) d \nu y d v x d \nu \\
& =\sum_{i} \int_{D_{i}} \int_{D_{i}^{c}} K(x, y) d \nu y d \nu x \\
& =\sum_{i} \int_{D_{i}} \sum_{j: i \neq j} \int_{D_{j}} K(x, y) d \nu y d \nu x+\sum_{i} \int_{D_{i}} \int_{D^{c}} K(x, y) d \nu y d v x \\
& =0+\int_{D} \int_{\mathbb{R}^{n} \backslash D} K(x, y) d v y d \nu x,
\end{aligned}
$$

where 0 comes from the antisymmetry of $K$. This gives

$$
\frac{1}{v(D)} \int_{D} S_{k+1} f d v=S_{k} f(z) \quad \text { for } z \in D
$$

and implies that $\left(S_{k} f, \mathcal{A}_{k}\right)$ is a martingale.

Now we check that the martingale $\left(S_{k} f, \mathcal{A}_{k}\right)$ is $L^{1}(v)$-bounded. Using 3.1), the Schwarz inequality and the $L^{2}$-boundedness of $T^{*}$, we estimate

$$
\begin{aligned}
\left|\int S_{k} f d \nu\right|= & \left|\sum_{D \in \mathcal{D}_{k}} \frac{1}{v(D)} \int_{D} \int_{D^{c}} K(x, y) d \nu y d v x v(D)\right| \\
= & \left|\sum_{D \in \mathcal{D}_{k}} \int_{D} \int_{D^{c}} K(x, y) f(y) d \mu y f(x) d \mu x\right| \\
\leq & \sum_{D \in \mathcal{D}_{k}}\left(\int_{D} T^{*}(f) f d \mu+\int_{D} T^{*}\left(f \chi_{D}\right) f d \mu\right) \\
\leq & \sum_{D \in \mathcal{D}_{k}}\left(\left(\int_{D} T^{*}(f)^{2} d \mu\right)^{1 / 2}\left(\int_{D} f^{2} d \mu\right)^{1 / 2}\right. \\
& \left.+\left(\int_{D} T^{*}\left(f \chi_{D}\right)^{2} d \mu\right)^{1 / 2}\left(\int_{D} f^{2} d \mu\right)^{1 / 2}\right) \\
\leq & \sum_{D \in \mathcal{D}_{k}}\left(\left(\int_{D} T^{*}(f)^{2} d \mu\right)^{1 / 2}+\left(C_{0} \int_{D} f^{2} d \mu\right)^{1 / 2}\right)\left(\int_{D} f^{2} d \mu\right)^{1 / 2}
\end{aligned}
$$




$$
\begin{aligned}
& \leq\left(\left(\sum_{D \in \mathcal{D}_{k}} \int_{D} T^{*}(f)^{2} d \mu\right)^{1 / 2}+\left(\sum_{D \in \mathcal{D}_{k}} C_{0} \int_{D} f^{2} d \mu\right)^{1 / 2}\right)\left(\sum_{D \in \mathcal{D}_{k}} \int_{D} f^{2} d \mu\right)^{1 / 2} \\
& =\left(\left(\int T^{*}(f)^{2} d \mu\right)^{1 / 2}+\left(C_{0} \int f^{2} d \mu\right)^{1 / 2}\right)\left(\int f^{2} d \mu\right)^{1 / 2} \leq 2 C_{0}^{1 / 2} \int f^{2} d \mu .
\end{aligned}
$$

This proves the $L^{1}$-boundedness. Hence by the martingale convergence theorem $\left(S_{k} f(z)\right)$ converges for $\mu$-almost all $z \in X$.

Now we also assume that

$$
\lim _{k \rightarrow \infty} \sup \left\{\operatorname{diam}(D): D \in \mathcal{D}_{k}\right\}=0 .
$$

For $f \in L^{2}(\mu)$ and $k \in \mathbb{N}$, we define

$$
A_{k} f(z)=\frac{1}{\mu(D)} \int_{D} \int_{D^{c}} K(x, y) f(y) d \mu y d \mu x \quad \text { when } z \in D \in \mathcal{D}_{k},
$$

where $A_{k} f(z)=0$ if $\mu(D)=0$. Using the convergence of $\left(S_{k} f(z)\right)$ we shall now verify that for $f \in L^{2}(\mu)$ the limit

$$
T f(z)=\lim _{k \rightarrow \infty} A_{k} f(z)
$$

exists and is finite for $\mu$-almost all $z \in X$. Clearly, we may assume that $f$ is non-negative. Moreover, since $A_{k}(f)=A_{k}(f+1)-A_{k}(1)$, we may assume that $f \geq 1$. To prove 3.3. for such an $f$, write $f_{D}=\frac{1}{\mu(D)} \int_{D} f d \mu$ for $D \in \mathcal{D}_{k}$ with $\mu(D)>0$. Then by [3.1], the Schwarz inequality and (1.5) we have, for $z \in D$,

$$
\begin{aligned}
& \left|S_{k} f(z)-A_{k} f(z)\right|=\left|\left(\int_{D} f d \mu\right)^{-1} \int_{D} \int_{D^{c}} K(x, y) f(y) d \mu y\left(f(x)-f_{D}\right) d \mu x\right| \\
& \quad \leq \frac{1}{\mu(D)}\left(\int_{D} T^{*}(f)\left|f-f_{D}\right| d \mu+\int_{D} T^{*}\left(f \chi_{D}\right)\left|f-f_{D}\right| d \mu\right) \\
& \quad \leq \frac{1}{\mu(D)}\left(\left(\int_{D} T^{*}(f)^{2} d \mu\right)^{1 / 2}+\left(\int_{D} T^{*}\left(f \chi_{D}\right)^{2} d \mu\right)^{1 / 2}\right)\left(\int_{D}\left(f-f_{D}\right)^{2} d \mu\right)^{1 / 2} \\
& \quad \leq\left(\frac{1}{\mu(D)}\left(2 \int_{D}\left(T^{*}(f)^{2}+C_{0} f^{2}\right) d \mu\right)^{1 / 2}\left(\frac{1}{\mu(D)} \int_{D}\left(f-f_{D}\right)^{2} d \mu\right)^{1 / 2}\right) .
\end{aligned}
$$

Here for $\mu$-almost all $z \in X$, as $k \rightarrow \infty$, the first factor goes to $2^{1 / 2}\left(T^{*}(f)(z)^{2}+\right.$ $\left.C_{0} f(z)^{2}\right)^{1 / 2}$, and the second goes to 0 . Hence $S_{k} f(z)-A_{k} f(z) \rightarrow 0$, which proves (3.3) for non-negative functions $f \in L^{2}(\mu)$ and of course then also for all $f \in L^{2}(\mu)$. Moreover, $T: L^{2}(\mu) \rightarrow L^{2}(\mu)$ is bounded.

To get from this the average convergence with balls one needs to approximate balls with nested systems. At least in $\mathbb{R}^{n}$ this approximation procedure can be done with dyadic cubes. The argument is quite technical and will be omitted. 


\section{Proof of Theorem 1.6}

We shall first make two reductions using the weak type inequality 1.11 . Firstly, we may assume that $f=1$. To see this, note that we may of course assume that $f$ is nonnegative. Bounded functions $f$ such that $f>\delta$ for some $\delta>0$ are dense in the space of non-negative $L^{1}(\mu)$-functions, whence standard techniques (as for 4.1 below) allow us to assume that $f$ is such a function. Replacing $\mu$ by $f \mu$ then gives the reduction to $f=1$.

Secondly, we may assume the uniform condition

$$
\mu(B(x, r)) \leq \eta(r) h(r) \leq h(r) \quad \text { for } x \in X, r>0,
$$

where $\eta$ is a non-decreasing function such that $\eta(r) \rightarrow 0$ as $r \rightarrow 0$. To see this, we use Egorov's theorem to select closed sets $E_{k}, k=1,2, \ldots$, such that $\mu\left(X \backslash E_{k}\right)<1 / k$ and $\mu(B(x, r)) / h(r) \rightarrow 0$ as $r \rightarrow 0$ uniformly on $E_{k}$. Then using (1.11) we have, for all $t>0$,

$$
\begin{aligned}
& \mu\left(\left\{x: \limsup _{\epsilon, \delta \rightarrow 0} \mid T_{\epsilon}(1)(x)\right.\right.\left.\left.-T_{\delta}(1)(x) \mid>t\right\}\right) \\
&= \mu\left(\left\{x: \limsup _{\epsilon, \delta \rightarrow 0} \mid T_{\epsilon}\left(1-\chi_{E_{k}}\right)(x)-T_{\delta}\left(1-\chi_{E_{k}}(x) \mid>t\right\}\right)\right. \\
& \leq \mu\left(\left\{x: T^{*}\left(1-\chi_{E_{k}}\right)(x)>t / 2\right\}\right) \leq C \mu\left(X \backslash E_{k}\right) / t,
\end{aligned}
$$

provided the $\operatorname{limit}_{\epsilon \rightarrow 0} \lim _{\epsilon}\left(\chi_{E_{k}}\right)(x)$ exists for $\mu$-almost all $x \in E_{k}$. (It also exists for all $x \in E_{k}^{c}$ since $E_{k}$ is closed.) That is, if we have the convergence for the measures $\chi_{E_{k}} \mu$, which satisfy (4.1), we have it also for $\mu$. Then it is easy to check that the limit must be $T(1)(x) \mu$-almost everywhere.

Thus it is enough to prove that $\lim _{\epsilon \rightarrow 0} T_{\epsilon}(1)(a)=T(1)(a)$ for $\mu$-almost all $a \in X$ assuming 4.1). It is enough to consider points $a \in X$ such that

$$
T(1)(a)=\lim _{\epsilon \rightarrow 0} \frac{1}{\mu(B(a, \epsilon))} \int_{B(a, \epsilon)} T(1) d \mu .
$$

Let $0<\delta<1 / 2$ and choose $p>1 / \delta$. Using 2.2) we can write, for $\epsilon>0$,

$$
\begin{aligned}
\phi(\epsilon):= & T_{\epsilon}(1)(a)-\frac{1}{\mu(B(a, \epsilon))} \int_{B(a, \epsilon)} T(1) d \mu \\
= & \int_{B(a, p \epsilon) \backslash B(a, \epsilon)} K(a, x) d \mu x \\
& +\left(\int_{B(a, p \epsilon)^{c}} K(a, x) d \mu x+\frac{1}{\mu(B(a, \epsilon))} \int_{B(a, p \epsilon)^{c}} T\left(\chi_{B(a, \epsilon)}\right) d \mu\right) \\
& +\frac{1}{\mu(B(a, \epsilon))} \int_{B(a, p \epsilon) \backslash B(a, \epsilon)} T\left(\chi_{B(a, \epsilon)} d \mu\right. \\
= & : \phi_{1}(\epsilon)+\phi_{2}(\epsilon)+\phi_{3}(\epsilon) .
\end{aligned}
$$


The first term is easy to estimate:

$$
\left|\phi_{1}(\epsilon)\right| \leq \frac{\mu(B(a, p \epsilon))}{h(\epsilon)} \leq C_{p} \frac{\mu(B(a, p \epsilon))}{h(p \epsilon)}<\delta
$$

by (1.7) and 1.10 ) for sufficiently small $\epsilon$. Here and later, $C_{q}$ for $q>1$ denotes a constant such that $h(q r) \leq C_{q} h(r)$ for $r>0$. We estimate $\phi_{2}$ using (1.8) and (4.1):

$$
\begin{aligned}
\left|\phi_{2}(\epsilon)\right| & =\left|\frac{1}{\mu(B(a, \epsilon))} \int_{B(a, \epsilon)}\left(\int_{B(a, p \epsilon)^{c}} K(a, x) d \mu x-\int_{B(a, p \epsilon)^{c}} K(y, x) d \mu x\right) d \mu y\right| \\
& \leq \frac{1}{\mu(B(a, \epsilon))} \int_{B(a, \epsilon)} \int_{B(a, p \epsilon)^{c}} \frac{d(a, y)}{d(a, x) h(d(a, x))} d \mu x d \mu y \\
& \leq \epsilon \sum_{i=0}^{\infty} \frac{\mu\left(B\left(a, 2^{i+1} p \epsilon\right)\right)}{2^{i} p \epsilon h\left(2^{i} p \epsilon\right)} \leq \sum_{i=0}^{\infty} \frac{\mu\left(B\left(a, 2^{i+1} p \epsilon\right)\right)}{2^{i} p C_{2}^{-1} h\left(2^{i+1} p \epsilon\right)} \leq 2 C_{2} / p<2 C_{2} \delta .
\end{aligned}
$$

To estimate $\phi_{3}$ we first show that at almost every point, $\mu$ is doubling at some small scales. Then we only need to treat the case $X=\mathbb{R}^{n}$. More precisely, let $C>2 C_{2}$ be a constant and let $F$ be the set of those $a \in \mathbb{R}^{n}$ for which there exists $\epsilon, 0<\epsilon<1$, such that

$$
\mu\left(B\left(a, 2^{1-k} \epsilon\right)\right) \geq C \mu\left(B\left(a, 2^{-k} \epsilon\right) \quad \text { for } k=0,1, \ldots\right.
$$

We also assume that $C>2^{n+1}$. We now show that $\mu(F)=0$. To prove this we may assume that the support of $\mu$ is bounded, say spt $\mu \subset B(0, R)$. For $a \in F$ let $\epsilon=\epsilon(a)$ be as above. Fix a large positive integer $m$ and pick for each $a \in F$ an integer $k(a) \geq m$ such that for $k \geq k(a)$,

$$
C^{-k} \leq\left(2^{-k} \epsilon(a)\right)^{n+1} .
$$

By Vitali's covering theorem (which holds in our setting, as we said at the beginning of the introduction) we can find disjoint balls $B\left(a_{i}, 2^{-k_{i}} \epsilon_{i}\right) \subset B(0, R)$ with $\epsilon_{i}=\epsilon\left(a_{i}\right)$ and $k_{i} \geq k\left(a_{i}\right)$ which cover $\mu$-almost all of $F$. Then

$$
\begin{aligned}
\mu(F) & \leq \sum_{i} \mu\left(B\left(a_{i}, 2^{-k_{i}} \epsilon_{i}\right)\right) \leq \sum_{i} C^{-k_{i}} \mu\left(B\left(a, \epsilon_{i}\right)\right) \\
& \leq \sum_{i}\left(2^{-k_{i}} \epsilon_{i}\right)^{n+1} \mu\left(\mathbb{R}^{n}\right) \leq R^{n} 2^{-m} \mu\left(\mathbb{R}^{n}\right) .
\end{aligned}
$$

Letting $m \rightarrow \infty$ we get $\mu(F)=0$.

Let now $a \in F^{c}$ and $0<\epsilon<1$. Then there is $k=0,1, \ldots$ such that

$$
\mu\left(B\left(a, 2^{1-k} \epsilon\right)\right) \leq C \mu\left(B\left(a, 2^{-k} \epsilon\right)\right)
$$

and

$$
\mu\left(B\left(a, 2^{1-j} \epsilon\right)\right) \geq C \mu\left(B\left(a, 2^{-j} \epsilon\right)\right) \quad \text { for } j=0, \ldots, k-1,
$$

whence

$$
\mu\left(B\left(a, 2^{-j} \epsilon\right)\right) \leq C^{-j} \mu(B(a, \epsilon)) \quad \text { for } j=0, \ldots, k-1
$$


Let $\epsilon_{1}=2^{-k} \epsilon$. Then $\mu\left(B\left(a, 2 \epsilon_{1}\right)\right) \leq C \mu\left(B\left(a, \epsilon_{1}\right)\right)$ and, since $C>2 C_{2}$, we get

$$
\begin{aligned}
\left|T_{\epsilon}(1)(a)-T_{\epsilon_{1}}(1)(a)\right| & \leq \sum_{j=1}^{k}\left|T_{2^{1-j} \epsilon}(1)(a)-T_{2^{-j} \epsilon}(1)(a)\right| \\
& \leq \sum_{j=1}^{k} \int_{B\left(a, 2^{1-j} \epsilon\right) \backslash B\left(a, 2^{-j} \epsilon\right)}|K(a, x)| d \mu x \leq \sum_{j=1}^{k} \frac{\mu\left(B\left(a, 2^{1-j} \epsilon\right)\right)}{h\left(2^{-j} \epsilon\right)} \\
& \leq \sum_{j=1}^{k} \frac{C^{1-j} \mu(B(a, \epsilon))}{C_{2}^{-j} h(\epsilon)} \leq C \eta(\epsilon) \sum_{j=1}^{k} 2^{-j} \leq C \eta(\epsilon)<\delta
\end{aligned}
$$

when $\epsilon$ is small enough. Consequently,

$$
\begin{aligned}
\left|\phi(\epsilon)-\phi\left(\epsilon_{1}\right)\right| \leq & \left|T_{\epsilon}(1)(a)-T_{\epsilon_{1}}(1)(a)\right| \\
& +\left|\frac{1}{\mu(B(a, \epsilon))} \int_{B(a, \epsilon)} T(1) d \mu-\frac{1}{\mu\left(B\left(a, \epsilon_{1}\right)\right)} \int_{B\left(a, \epsilon_{1}\right)} T(1) d \mu\right|<\delta
\end{aligned}
$$

when $\epsilon$ is small enough. Now we estimate the average of $\left|\phi_{3}(t)\right|$ over $\left[\epsilon_{1}, 2 \epsilon_{1}\right]$ by

$$
\begin{aligned}
\frac{1}{\epsilon_{1}} \int_{\epsilon_{1}}^{2 \epsilon_{1}}\left|\phi_{3}(t)\right| d t & \leq \frac{1}{\epsilon_{1}} \int_{\epsilon_{1}}^{2 \epsilon_{1}} \frac{1}{\mu(B(a, t))} \int_{B(a, p t) \backslash B(a, t)} \int_{B(a, t)}|K(x, y)| d \mu x d \mu y d t \\
& =\frac{1}{\epsilon_{1}} \iiint_{A} \frac{1}{\mu(B(a, t))}|K(x, y)| d \mu x d \mu y d t
\end{aligned}
$$

where

$$
\begin{aligned}
A & =\left\{(x, y, t): d(x, a)<t \leq d(y, a)<p t, \epsilon_{1} \leq t \leq 2 \epsilon_{1}\right\} \\
& \subset\left\{(x, y, t): d(x, a)<2 \epsilon_{1}, d(y, a)<2 p \epsilon_{1}, d(x, a)<t \leq d(y, a)\right\} .
\end{aligned}
$$

Thus by Fubini's theorem, 1.7 and 4.1,

$$
\begin{aligned}
& \frac{1}{\epsilon_{1}} \int_{\epsilon_{1}}^{2 \epsilon_{1}}\left|\phi_{3}(t)\right| d t \leq \frac{1}{\epsilon_{1} \mu\left(B\left(a, \epsilon_{1}\right)\right)} \int_{B\left(a, 2 p \epsilon_{1}\right)} \int_{B\left(a, 2 \epsilon_{1}\right)}|K(x, y)| \int_{d(x, a)}^{d(y, a)} d t d \mu x d \mu y \\
& =\frac{1}{\epsilon_{1} \mu\left(B\left(a, \epsilon_{1}\right)\right)} \int_{B\left(a, 2 \epsilon_{1}\right)} \int_{B\left(a, 2 p \epsilon_{1}\right)}|K(x, y)|(d(y, a)-d(x, a)) d \mu y d \mu x \\
& \leq \frac{1}{\epsilon_{1} \mu\left(B\left(a, \epsilon_{1}\right)\right)} \int_{B\left(a, 2 \epsilon_{1}\right)} \int_{B\left(x, 2(p+1) \epsilon_{1}\right)}|K(x, y)| d(x, y) d \mu y d \mu x \\
& \leq \frac{1}{\epsilon_{1} \mu\left(B\left(a, \epsilon_{1}\right)\right)} \int_{B\left(a, 2 \epsilon_{1}\right)} \sum_{i=0}^{\infty} \int_{B\left(x, 2^{1-i}(p+1) \epsilon_{1}\right) \backslash B\left(x, 2^{-i}(p+1) \epsilon_{1}\right)}|K(x, y)| d(x, y) d \mu y d \mu x \\
& \leq \frac{1}{\epsilon_{1} \mu\left(B\left(a, \epsilon_{1}\right)\right)} \int_{B\left(a, 2 \epsilon_{1}\right)} \sum_{i=0}^{\infty} \frac{2^{1-i}(p+1) \epsilon_{1} \mu\left(B\left(x, 2^{1-i}(p+1) \epsilon_{1}\right)\right)}{h\left(2^{-i}(p+1) \epsilon_{1}\right)} d \mu x
\end{aligned}
$$




$$
\begin{aligned}
& \leq \frac{1}{\epsilon_{1} \mu\left(B\left(a, \epsilon_{1}\right)\right)} \sum_{i=0}^{\infty} \frac{2^{1-i}(p+1) \epsilon_{1} \eta\left(2^{1-i}(p+1) \epsilon_{1}\right) h\left(2^{1-i}(p+1) \epsilon_{1}\right)}{h\left(2^{-i}(p+1) \epsilon_{1}\right)} \mu\left(B\left(a, 2 \epsilon_{1}\right)\right) \\
& \leq \frac{4 C_{2}(p+1) \eta\left(2(p+1) \epsilon_{1}\right) \mu\left(B\left(a, 2 \epsilon_{1}\right)\right)}{\mu\left(B\left(a, \epsilon_{1}\right)\right)} \leq 4 C C_{2}(p+1) \eta(2(p+1) \epsilon)<\delta
\end{aligned}
$$

when $\epsilon$ is small enough. So there is $\epsilon_{2}$ with $\epsilon_{1} \leq \epsilon_{2} \leq 2 \epsilon_{1}$ such that $\left|\phi_{3}\left(\epsilon_{2}\right)\right|<\delta$. Then $\left|\phi\left(\epsilon_{1}\right)-\phi\left(\epsilon_{2}\right)\right|<\delta$ as above and so

$$
\begin{aligned}
|\phi(\epsilon)| & \leq\left|\phi(\epsilon)-\phi\left(\epsilon_{1}\right)\right|+\left|\phi\left(\epsilon_{1}\right)-\phi\left(\epsilon_{2}\right)\right|+\left|\phi\left(\epsilon_{2}\right)\right| \\
& <2 \delta+\left|\phi_{1}\left(\epsilon_{2}\right)\right|+\left|\phi_{2}\left(\epsilon_{2}\right)\right|+\left|\phi_{3}\left(\epsilon_{2}\right)\right|<\left(4+2 C_{2}\right) \delta .
\end{aligned}
$$

This completes the proof of Theorem 1.6.

\section{Remarks on rectifiability}

One motivation for the developments in this paper was to find some new insight into the following problem:

Let $m$ be an integer, $0<m<n$, and let $\mu$ be an $m$-dimensional Ahlfors-Davidregular Borel measure on $\mathbb{R}^{n}$, as in Section 1 . For $i=1, \ldots, n$ let $T_{i}^{*}$ be the maximal operator related to $\mu$ and the kernel $|x-y|^{-m-1}\left(x_{i}-y_{i}\right)$. Suppose that each $T_{i}^{*}$ is bounded in $L^{2}(\mu)$. Does $\mu$ have to be rectifiable, or even uniformly rectifiable in the sense of David and Semmes?

By the rectifiability of $\mu$ we mean that there are $m$-dimensional $C^{1}$-surfaces $M_{1}$, $M_{2}, \ldots$ such that $\mu\left(\mathbb{R}^{n} \backslash \bigcup_{i} M_{i}\right)=0$. For the definitions of uniform rectifiability, see [DS].

If $m=1$, the answer to the above question is yes by [MMV], and the regularity assumptions on $\mu$ can be considerably relaxed (see [T2]). The problem is open for $m \geq 2$.

It was shown in [MPr] (see also [M]) that the rectifiability of an Ahlfors-Davidregular measure $\mu$ follows from the existence of the principal values

$$
\lim _{\epsilon \rightarrow 0} \int_{\mathbb{R}^{n} \backslash B(x, \epsilon)}|x-y|^{-m-1}\left(x_{i}-y_{i}\right) d \mu y, \quad i=1, \ldots, n,
$$

for $\mu$-almost all $x \in \mathbb{R}^{n}$. The Ahlfors-David regularity assumption has recently been considerably relaxed by Tolsa [T4]. But it is not known whether the $L^{2}$-boundedness implies the above almost everywhere convergence. Thus Theorem 1.4 is a kind of replacement for this. Unfortunately we do not know if the almost everywhere convergence of the averages in Theorem 1.4 implies rectifiability, nor do we know whether it implies the almost everywhere existence of the principal values in this particular case.

These questions are also related to geometric properties of removable sets of bounded analytic functions in $\mathbb{C}$ (see [MMV], [P] and [T3]) and of Lipschitz harmonic functions in $\mathbb{R}^{n}$ (see $[\overline{\mathrm{MP}}]$ ).

The $L^{2}$-boundedness does not always imply the almost everywhere existence of principal values in the setting of Theorem 1.4. This can be seen by considering a standard 
example of a purely unrectifiable 1-dimensional Ahlfors-David-regular set in the plane, which is the Cantor set obtained by starting with the unit square, taking four squares of side-length $1 / 4$ inside it in its corners, then taking the squares of side-length $1 / 16$ in the corners of these, and so on. The final Cantor set $C$ is the compact set inside all these squares of all generations. In [D2] David constructed a 1-dimensional odd CalderónZygmund kernel $K$ such that the operator $T^{*}$ related to $K$ is bounded in $L^{2}(\mu)$ where $\mu$ is the natural (1-dimensional Hausdorff) measure on $C$. However, it is easy to check that the principal values

$$
\lim _{\epsilon \rightarrow 0} \int_{B(x, \epsilon)^{c}} K(x-y) d \mu y
$$

fail to exist at $\mu$-almost all points $x \in \mathbb{R}^{2}$. New interesting examples of non-existence of principal values under $L^{2}$-boundedness have been constructed by Chousionis [Ch].

In [H2] Huovinen considered homogeneous kernels such as

$$
K(z)=\operatorname{Re}\left(z /|z|^{2}-z^{3} /|z|^{4}\right)
$$

for $z \in \mathbb{C}$. He showed that there exist purely unrectifiable 1-dimensional Ahlfors-Davidregular sets on which for such a kernel the principal values exist almost everywhere and the related operator is bounded in $L^{2}$ on some subset of positive measure. On the other hand, he showed in [H1] that for the kernels $z^{2 k-1} /|z|^{2 k}, k=1,2, \ldots$, and their linear combinations the almost everywhere convergence of principal values on 1-dimensional AD-regular sets implies their rectifiability.

Acknowledgments. The authors are grateful to the "Centre de Recerca Matemàtica" for the perfect organization of a special trimester in Fourier Analysis during the spring of 2006. The event offered an excellent opportunity to work on the paper. The second named author was partially supported by grants 2005SGR00774 (Generalitat de Catalunya), MTM2004-00519 and HF2004-0208.

\section{References}

[Ch] Chousionis, V.: Singular integrals on Sierpinski gaskets. Publ. Mat. 53, 245-256 (2009)

[C] Christ, M.: Lectures on Singular Integral Operators. CBMS Reg. Conf. Ser. Math. 77, Amer. Math. Soc. (1990) Zbl 0745.42008 MR 1104656

[CW] Coifman, R. R., Weiss, G.: Analyse harmonique non-commutative sur certains espaces homogènes. Lecture Notes in Math. 242, Springer (1971) Zbl 0224.43006 MR 0499948

[D1] David, G.: Wavelets and Singular Integrals on Curves and Surfaces. Lecture Notes in Math. 1465, Springer (1991) Zbl 0764.42019 MR 1123480

[D2] David, G.: Des intégrales singulières sur un ensemble de Cantor. C. R. Acad. Sci. Paris Sér. I Math. 332, 391-396 (2001) Zbl 1047.42006 MR 1826622

[DS] David, G., Semmes, S.: Analysis of and on Uniformly Rectifiable Sets. Math. Surveys Monogr. 38, Amer. Math. Soc. (1993) Zbl 0832.42008 MR 1251061

[F] Federer, H.: Geometric Measure Theory. Springer (1969) Zbl 0176.00801 MR 0257325

[H1] Huovinen, P.: Singular integrals and rectifiability of measures in the plane. Ann. Acad. Sci. Fenn. Math. Diss. 109, 1-63 (1997) Zbl 0883.28007 MR 1428746

[H2] Huovinen, P.: A nicely behaved singular integral on a purely unrectifiable set. Proc. Amer. Math. Soc. 129, 3345-3351 (2001) Zbl 0973.42007 MR 1845012 
[M] Mattila, P.: Geometry of Sets and Measures in Euclidean Spaces. Cambridge Univ. Press (1995) Zbl 0819.28004 MR 1333890

[MMV] Mattila, P., Melnikov, M. S., Verdera, J.: The Cauchy integral, analytic capacity, and uniform rectifiability. Ann. of Math. 144, 127-136 (1996) Zbl 0897.42007 MR 1405945

[MP] Mattila, P., Paramonov, P. V.: On geometric properties of harmonic Lip ${ }_{1}$-capacity. Pacific J. Math. 171, 469-491 (1995) Zbl 0852.31004 MR 1372240

[MPr] Mattila, P., Preiss, D.: Rectifiable measures in $\mathbb{R}^{n}$ and existence of principal values for singular integrals. J. London Math. Soc. 52, 482-496 (1994) Zbl 0880.28002 MR 1363815

[NTV] Nazarov, F., Treil, S., Volberg, A.: Weak type estimates and Cotlar inequalities for Calderón-Zygmund operators on nonhomogeneous spaces, Int. Math. Res. Notices 1998, no. 9, 463-487 Zbl 0918.42009 MR 1626935

[P] Pajot, H.: Analytic Capacity, Rectifiability, Menger Curvature and the Cauchy Integral. Lecture Notes in Math. 1799, Springer (2002) Zbl 1043.28002 MR 1952175

[T1] Tolsa, X.: Cotlar's inequality and the existence of principal values for the Cauchy integral without doubling condition. J. Reine Angew. Math. 502, 199-235 (1998) Zbl 0912.42009 MR 1647575

[T2] Tolsa, X.: Principal values for the Cauchy integral and rectifiability. Proc. Amer. Math. Soc. 128, 2111-2119 (2000) Zbl 0944.30022 MR 1654076

[T3] Tolsa, X.: Painlevé's problem and the semiadditivity of the analytic capacity. Acta Math. 190, 105-149 (2003) Zbl 1060.30031 MR 1982794

[T4] Tolsa, X.: Principal values for Riesz transforms and rectifiability. J. Funct. Anal. 254, 1811-1863 (2008)

[Ve] Verdera, J.: The fall of the doubling condition in Calderón-Zygmund theory. In: Proceedings of the 6th International Conference on Harmonic Analysis and Partial Differential Equations (El Escorial, 2000), Publ. Mat. 2002, Vol. Extra, 275-292 Zbl 1025.42008 MR 1964824

[V] Volberg, A.: Calderón-Zygmund Capacities and Operators on Nonhomogeneous Spaces. CBMS Reg. Conf. Ser. Math. 100, Amer. Math. Soc. (2002) Zbl 1053.42022 MR 2019058 